\title{
El índice de Malmquist y los efectos de la educación superior sobre la productividad nacional
}

\section{The Malmquist Index and the effects of higher education on national productivity}

DOI: https://doi.org/10.32870/dse.v0i22.707

María Fernanda Higuera Cota*

Santos López Leyva**

Óscar Omar Cruz Castro***

\begin{abstract}
Resumen
Las instituciones de educación superior representan un elemento clave para el desarrollo de los individuos, mismo que se ve reflejado en el desarrollo económico y social de los países. Este trabajo da a conocer específicamente en qué ayuda la educación superior en determinadas áreas, y pone de manifiesto que problemas como la falta de inversión en este sector frenan la productividad y el interés de investigar en el área. Lo anterior se cumple mediante la implementación del índice de Malmquist, al establecer comparaciones entre los 43 países que poseen universidades catalogadas dentro del World University Ranking para los años 2012, 2013 y 2014, y la formulación de un punto de referencia para ayudar a detectar qué áreas son oportunidades para mejorar y alcanzar mayor productividad.
\end{abstract}

Palabras clave: educación superior - productividad - desarrollo económico.

\begin{abstract}
Higher education institutions represent a key element for the development of individuals, which is reflected in the economic and social development of countries. This study reveals how higher education in particular helps in certain areas, and shows that problems such as lack of investment in this sector slow down productivity and the interest in researching in the area through the implementation of the Malmquist index, comparing the 43 countries that have universities cataloged within the World University Ranking for 2012, 2013 and 2014, and formulating a benchmark to help detect which areas provide opportunities to improve and achieve higher productivity.
\end{abstract}

Key words: higher education - productivity - economic development.

\footnotetext{
* Doctora en Estudios del Desarrollo Global. Profesora de asignatura en la Facultad de Administración y Contabilidad, Universidad Autónoma de Baja California. México. higuera.maria@uabc.edu.mx

** Doctor en Economía. Profesor-investigador en la Facultad de Economía y Relaciones Internacionales (FEYRI) de la Universidad Autónoma de Baja California. México. Sanlop1947@gmail.com

*** Doctorante en Ciencias Económicas. FEYRI de la Universidad Autónoma de Baja California, México. ocruz@uacb.edu.mx
} 


\section{Introducción}

En este trabajo se analiza la Productividad Total de Factores (PTF) en 43 países, los cuales cuentan con universidades catalogadas dentro del World University Ranking (ARWU) y el efecto que tiene la educación superior en dicha PTF, para los periodos 2012, 2013 y 2014, haciendo uso de datos provenientes del ARWU y el Banco Mundial. Las estimaciones se realizan mediante una metodología no paramétrica, basada en el Análisis Envolvente de Datos (DEA por sus siglas en inglés), con el objetivo de procesar los datos para dar como resultado el índice de Malmquist, el cual refleja los cambios en PTF. Esta metodología posibilita la descomposición de los cambios en la PTF en un componente de eficiencia y en otro de cambio tecnológico. Dichos cambios son denominados "catch up", es decir, el acercamiento hacia la frontera tecnológica de innovación. ${ }^{1}$ Asimismo, la metodología permite conocer los cambios en la eficiencia pura y en cambios de escala.

Se parte del cuestionamiento: ¿Cuáles son los efectos de la educación superior sobre la PTF de los 43 países seleccionados? Se puede apreciar que el objetivo de este trabajo es evaluar el efecto de eficiencia y el efecto de frontera que experimentan estos países en relación con la educación superior, para así conocer el nivel de PTF de cada país, e inferir en qué medida la educación superior es o no un elemento clave al momento de medir la PTF de los países.

La utilización del índice de productividad de Malmquist (1953) permite conocer el crecimiento de la productividad total de los factores de cada país. Como resultado de dicho análisis, se esperaría encontrar que los países que posean universidades posicionadas en las primeras clasificaciones del ARWU sean también los que experimenten niveles más altos de PTF.

\section{Marco teórico}

\section{El rol de la educación superior}

Para partir del concepto de educación, Kantor (1978) explica que la educación es un proceso de socialización planificada ya que facilita el desarrollo de la inteligencia y el aprendizaje. A su vez, postula que la educación debe ser entendida como el establecimiento de actividades funcionales de una manera planificada, para que el aprendizaje sea considerado como capacidad intelectual, al enseñar cómo solucionar problemas.

Así pues, la educación es un proceso humano y cultural difícil de comprender. Para poder entenderla se debe partir de su propósito e interpretación. Para el logro de lo anterior es necesario considerar el entorno del hombre, ya que es un ser social que a lo largo de su vida establece vínculos y relaciones de interdependencia con otros seres humanos. Es por ello que la educación no puede definirse sin estudiar antes la cultura, la sociedad y el aprendizaje.

León (2007) sostiene que la cultura es todo aquello que el hombre ha creado apoyándose en lo que la naturaleza le ha provisto para ello. Asimismo, considera la creación como algo indi-

1Frontera tecnológica es el conjunto de elementos que generan efectos directos sobre la productividad, y a su vez están vinculados con el volumen de comercio (Griffith et al., 2004).

Diálo@os 
vidual y colectivo a la vez. Al hablar de creaciones, el autor menciona a la tecnología, la religión, la ciencia, los mitos, las artes, los lenguajes, las costumbres, la moral, las formas de pensar y de hacer, simbolismos, significados, etcétera.

En cambio, en 1871 Tylor (Grimson, 2008) planteó un concepto de cultura asociado a todo aquello que se relacionara con conocimientos, tradición, costumbres y hábitos inherentes a la persona dentro de una sociedad, al ser perteneciente a esta.

Al igual que educación, sociedad es un término complejo, pues al hablar de sociedad se puede estar partiendo de realidades distintas. Giddens (1999) la define como "el grupo de personas que vive en un territorio determinado, sometido a un sistema común de autoridad política y que es consciente de poseer una identidad que lo distingue de otros grupos". Mientras que para Falicov y Lifzyc (2002: 187) "la sociedad tiene una existencia propia que va más allá de la experiencia personal... porque desde antes del nacimiento de cada individuo existen formas reiteradas y consideradas correctas de comportamiento que se van transmitiendo de generación en generación".

Asimismo, la Real Academia de la Lengua española describe el aprendizaje como el proceso por el cual las personas adquieren cambios en su comportamiento, mejoran sus actuaciones, reorganizan su pensamiento o descubren nuevas maneras de comportamiento y nuevos conceptos e información.

Figura 1. Educación integral

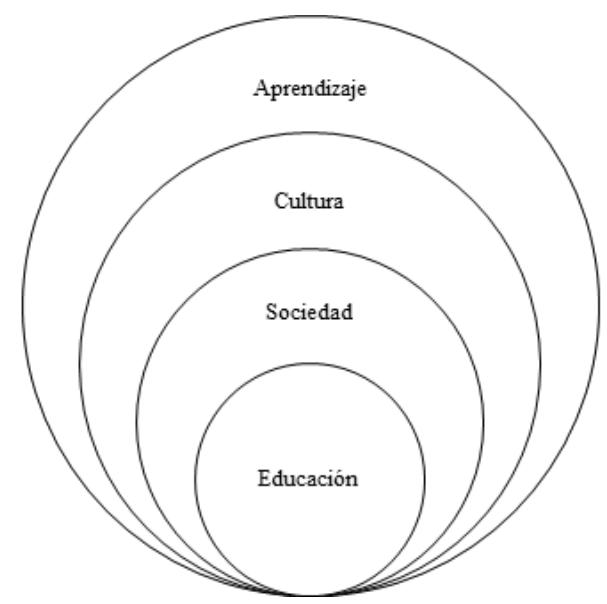

Fuente: elaboración propia con información de Kantor (1978), León (2007), Grimson (2008), Giddens (1999), Falicov y Lifzyc (2002), Real Academia Española (2018).

En la figura 1 se muestra el vínculo existente entre educación, sociedad, cultura y aprendizaje. Se busca destacar la relación entre los conceptos y la latente necesidad de una adecuada 
dirección de cada uno de ellos, para el logro de una educación integral. Se parte de la aportación de Kantor (1978), quien considera la educación como un proceso de socialización y planificación; es ahí donde tiene lugar el concepto de aprendizaje, en el entendido de que este hace referencia a un proceso que produce cambios de comportamiento. Conviene subrayar que en la acepción general de educación se hace alusión directamente al aprendizaje, considerada como un proceso que facilita el desarrollo y el aprendizaje.

En este contexto, León (2007) sostiene que la educación es un proceso humano y cultural, haciendo así una referencia directa a la cultura, y destaca que, para que este proceso humano surja, son necesarias las creaciones personales y colectivas, mediante conocimientos, tradiciones, costumbres y hábitos dentro de una sociedad. Es aquí donde se encuentra la conexión entre los términos ya que, para que cada uno de ellos se presente de manera exitosa, es necesario que el individuo se encuentre en un ambiente de socialización sano, donde "los seres sean conscientes de poseer una identidad y se sientan pertenecientes a un sistema político" (Giddens, 1999: 739), puesto que es a través de este sistema que se crean los planes y programas que dictan las directrices que se han de seguir para el logro de las competencias y los objetivos de la educación.

También, Falicov y Lifzyc (2002) afirman que la sociedad va más allá de la experiencia personal, porque prexisten formas reiteradas y consideradas correctas de comportamiento, en donde se puede observar nuevamente el enlace con cultura, entendida como el conjunto de hábitos inherentes a una sociedad, en el cual se manifiestan conocimientos, tradiciones y costumbres (Tylor, 1871).

El análisis anterior promueve un acercamiento con el papel de la educación en la sociedad, bajo el supuesto de que una eficiente gestión de la educación en el país permitirá alcanzar mayores niveles de desarrollo y, con ello, mejorar la calidad de vida de la población. No obstante, si se desea conocer la productividad de un país, es necesario reflexionar acerca del papel de la educación terciaria. De acuerdo con la UNESCO (2011), en la Clasificación Internacional Normalizada de la Educación (CINE), la educación terciaria o nivel equivalente corresponde al CINE 6, donde la duración de los programas del nivel puede variar entre tres y cuatro años a partir del nivel CINE $3^{2}$, o entre uno y dos años a partir de otro programa del nivel CINE 6.

\section{La educación superior como medida de desarrollo}

Con los cambios experimentados en el contexto mundial han surgido nuevos escenarios (transformaciones en usos y costumbres en el mercado laboral, mercado financiero, mercado de consumidores, sistema político, educación, etc.) donde los individuos tienen que desempeñarse

2 La edad típica de ingreso al nivel CINE 3 fluctúa entre los 14 y 16 años. “Los programas comprendidos en este nivel suelen tener una duración de 12 o 13 años a partir del inicio del nivel CINE 1 (es decir, cuando el estudiante tiene aproximadamente 17 o 18 años de edad)" (UNESCO, 2011).

Diálo pos 
de manera competitiva ${ }^{3}$ para lograr seguir dentro de las dinámicas económicas y no verse desplazados. Ya lo mencionaba Beck (1998: 97): "Se está llevando a cabo una revolución cultural y social como consecuencia de la globalización económica".

En relación con las variaciones que experimentó la educación, esta comenzó a tener relevancia en la agenda internacional en 1990, en el marco de la Conferencia de Jomtien y su Declaración Mundial sobre Educación para Todos (CEPAL, 2002). Lo cual vino a romper los esquemas de la educación percibidos y representó un punto de referencia para la reorientación de políticas educativas para la trasformación de los sistemas de enseñanza a nivel mundial. Dichos sistemas deben contener planes y programas que permitan universalizar la calidad de la oferta educativa, fomentar la inclusión y formar al ciudadano para la convivencia y el aprendizaje a lo largo de su vida.

Así pues, la sociedad del conocimiento, como la denomina Delors (1996), no limita el aprendizaje a los espacios institucionales, sino que promueve que este se dé en todos los contextos. Por otro lado, García (2006) señala que el aprendizaje no puede quedar limitado a un periodo temporal en el ciclo vital de la persona, y que los años de formación no son suficientes puesto que el sujeto se ve obligado a aprender a lo largo de toda su vida. Lo anterior, debido a los continuos cambios en todos los niveles y a que estos conllevan nuevas demandas profesionales y nuevas exigencias personales.

Asimismo, en la sociedad del conocimiento cada individuo debe ser capaz de adaptarse a los contextos mediante la asimilación de una base de conocimientos rigurosos y estrategias eficaces, con el único objetivo de actuar acertadamente ante ciertas situaciones a lo largo de su existencia; para el logro de lo anterior, tanto Delors (1996) como García (2006) proponen hacerlo desde criterios razonables y susceptibles de crítica, el desarrollo del pensamiento reflexivo, crítico y creativo, para asegurar con ello una sensibilidad mayor a las exigencias cambiantes de los contextos o dinámicas actuales. Es así que se llega a la conclusión de que la educación superior es un elemento clave para el desarrollo de las sociedades.

\section{El papel de la productividad en el desarrollo económico y social}

Se eligió estudiar la productividad de los países debido a la cercanía que esta guarda con el estándar de vida de una nación o calidad de vida. ${ }^{4}$ Morales y Masis (2014), Carro y González (2014), así como el INEGI (1996), reconocen que esta se encuentra asociada a la producción, y que su estudio surgió con la revolución industrial: "en 1948, se crea el Consejo Productivo

3 Competitividad es la capacidad que posee una persona, empresa o país para generar rentabilidad sobre la competencia.

4 Ardila define la calidad de vida como "un estado de satisfacción general, derivado de la realización de las potencialidades de la persona. Posee aspectos subjetivos y aspectos objetivos. Es una sensación subjetiva de bienestar físico, psicológico y social. Incluye aspectos objetivos como el bienestar material, las relaciones armónicas con el ambiente físico y social y con la comunidad, y la salud objetivamente percibida" (Ardila, 2003). 
Anglo-Americano, que sirvió de base entre la Europa devastada por la guerra y Estados Unidos victoriosos y posicionados como potencia económica y militar. Por otro lado, Japón experimentó, como el ave fénix, el resurgimiento del problema de productividad cuando estudió las bases de dicho consejo" (Morales, Masis, 2014: 42).

"Según una definición general, la productividad es la relación entre la producción obtenida por un sistema de producción obtenida por un sistema de producción o servicios y los recursos utilizados para obtenerla" (Prokopenko, 1989: 3). A su vez, Prokopenko describe la productividad como el uso eficiente de recursos (tierra, capital, trabajo, materiales, información, etc.) en la producción de diversos bienes y servicios. Para Carro y González, la productividad implica la mejora del proceso productivo, tal como señalan textualmente:"la mejora significa una comparación favorable entre la cantidad de recursos utilizados y la cantidad de bienes utilizados y la cantidad de bienes y servicios producidos" (2012: 1).

\section{Productividad $=$ imputs $/$ outputs}

Basta analizar la fórmula anterior para encontrar que surge una serie de imposiciones para comprenderla en su totalidad, pues es necesario definir el sistema, el cual, para efectos de este artículo, son los países; lo mismo sucede con la definición de los inputs y outputs utilizados para la medición de la productividad.

La relevancia de estudiar la productividad viene del hecho de que su crecimiento permite medir el uso eficiente de los recursos con los que cuenta una empresa, un sector o un país. Tal como se describe en la publicación el ABC de la Productividad en 1996, "la productividad desempeña un papel central en el crecimiento económico que puede alcanzar un país, hecho que pone de manifiesto la importancia de avanzar en un mayor conocimiento de esta".

Existen una serie de elementos que se deben comprender al momento de profundizar en la productividad, mismos que se encuentran en la figura 2.

Diálo@os 
Figura 2. Elementos de la productividad

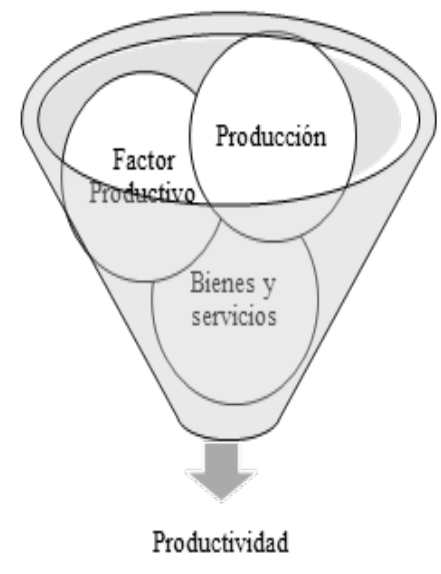

Elaboración propia con información del INEGI (1996).

Tal como se puede observar en la figura anterior, para hacer posible el estudio del concepto de productividad es necesario conocer cuáles son las implicaciones del proceso productivo; para ello, es necesario definir qué es producción, cuáles son los factores productivos y a qué se le considera un bien y un servicio.

Para responder el primer cuestionamiento, el INEGI (1996: 7) sostiene que "es la actividad en la cual los factores de producción se combinan y transforman en bienes y servicios". De ahí la importancia de la cantidad y calidad de los factores de producción que intervienen en el proceso productivo. Mientras que el factor productivo comprende inicialmente a la tierra, el capital y el trabajo, en recientes estudios incluye a la tecnología y el conocimiento. Por último, en economía, los bienes (físicos) y los servicios (intangibles) corresponden al resultado de los esfuerzos para satisfacer necesidades y deseos de las personas.

Partiendo de la definición de productividad como la "relación entre insumo y producto" (Mertens, 1999: 7), del papel que desempeña la educación en el desarrollo de un país, así como de la inferencia de que los países operan como empresas al gestionar recursos para la obtención de rendimientos mayores, se percibe a la educación, como una de las formas para incrementarlos y al ser una inversión, se convierte en un componente clave de la productividad individual y de la sociedad desde el momento en que la persona es consciente de que se encuentra dentro de un sistema político y desempeña un rol dentro de este. La educación aumenta el tiempo de vida útil del individuo al no depender únicamente de la fuerza de trabajo. Además, diversifica las áreas de empleo, incrementando con ello las oportunidades de colocación dentro del mercado laboral.

Por otra parte, la educación ayuda a tener mejor salud y constituye un acervo cultural mayor, y al ser considerada como el derecho que permite ejercer los demás derechos (UNICEF, 
2000), promueve un aumento de la democracia puesto que forma individuos cada vez más conscientes de lo que sucede en su entorno.

\section{Análisis de variables e indicadores}

\section{Composición del Academic Ranking of World Universities (ARWU)}

El Academic Ranking of World Universities, es un ranking mundial de universidades que utiliza

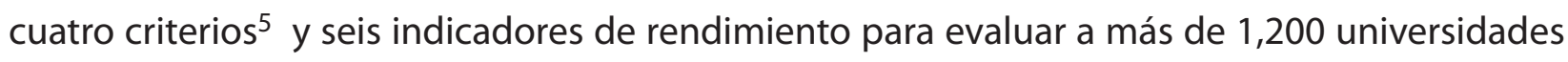
desde 2003, de las cuales las mejores 500 son seleccionadas y clasificadas entre las 20, 100, 200, 300,400 y 500 más sobresalientes. A continuación, se muestra una tabla con los indicadores de rendimiento.

Tabla 1. Indicadores de rendimiento del Academic Ranking of World Universities

\begin{tabular}{|c|c|c|}
\hline Indicador & Código & Puntaje \\
\hline $\begin{array}{l}\text { 1) Número total de exalumnos de una institución que ganó Premios Nobel y } \\
\text { Medallas Fields. Se definen como aquellos sujetos que obtienen títulos ya sea } \\
\text { de licenciatura, maestría o doctorado de la institución. }\end{array}$ & Alumni & $10 \%$ \\
\hline $\begin{array}{l}\text { 2) Número total de personal de una institución que ganó los premios Nobel en } \\
\text { Física, Química, Medicina y Medalla de Campos en Matemáticas. Es el conjunto } \\
\text { de personas que trabajan en la institución al momento de ganar el premio. }\end{array}$ & Award & $20 \%$ \\
\hline $\begin{array}{l}\text { 3) Número de investigadores altamente citados, seleccionados por Thomson } \\
\text { Reuters. Se obtiene a partir de una lista de investigadores denominada HCR } \\
2015 \text {. }\end{array}$ & $\mathrm{HiCi}$ & $20 \%$ \\
\hline $\begin{array}{l}\text { 4) Número de trabajos publicados en Nature and Science. Se le otorga un por- } \\
\text { centaje a cada afiliación de autor que va de } 10 \text { a } 100 \% \text {, dependiendo del tipo de } \\
\text { participación.* }\end{array}$ & $N \& S$ & $20 \%$ \\
\hline $\begin{array}{l}\text { 5) Número total de artículos indexados en Science Citation Index-Expand y So- } \\
\text { cial Science Citation Index. En este apartado solo se consideran las publicaciones } \\
\text { tipo artículo al calcular el número total de documentos de una institución. }\end{array}$ & PUB & $20 \%$ \\
\hline \multirow[t]{2}{*}{$\begin{array}{l}\text { 6) Las puntuaciones ponderadas de los cinco indicadores anteriores se dividen } \\
\text { entre la cantidad de personal académico equivalente a tiempo completo. }\end{array}$} & PCP & $20 \%$ \\
\hline & Total & $100 \%$ \\
\hline
\end{tabular}

Fuente: elaboración propia con información del ARWU (2017).

Tal como se observa en la tabla anterior, a cada indicador se le proporciona un puntaje ponderado que suma $100 \%$, es decir, se agrupan los puntajes a fin de conseguir un puntaje general, mismo que solo es asignado a la institución mayor; en el resto de las universidades se calculan con un porcentaje del puntaje máximo.

5 Los criterios para evaluar a las universidades del mundo según el ARWU (2017) son: calidad de la educación, calidad de la facultad, resultados de investigación y actuación per cápita.

* Afiliación de autor correspondiente a 100\%, afiliación de primer autor 50\%, segunda afiliación 25\% y 10\% para otras afiliaciones.

Diálo@os 
Pero, ¿cuál es la importancia de los rankings mundiales de universidades? Villaseñor, Moreno y Flores (2015) señalan que, más allá de constituir un fenómeno reciente derivado de la globalización, es una señal inequívoca de que las universidades viven realmente en una época de mediciones y comparaciones. A su vez, los autores hacen un análisis en el que concluyen que el éxito de los rankings se fundamenta en su pretendido valor informativo para la toma de decisiones. Además, distinguen que numerosos líderes institucionales hacen uso de estos rankings universitarios como instrumentos para el diseño de planes y políticas de sus instituciones.

Por otro lado, Salmi y Saroyan (2007) destacan que en un principio los rankings se pensaron con la finalidad de comparar el desempeño de unas universidades con otras. Lo cual se traduce en una búsqueda continua de la excelencia y mejora del desempeño y la productividad de las universidades mediante la competencia.

En la siguiente tabla se muestran los indicadores de entrada y salida utilizados para medir los efectos de la educación superior en la productividad.

Tabla 2. Indicadores de educación superior y PTF

\begin{tabular}{|l|l|}
\hline \multicolumn{1}{|c|}{ Inputs } & \multicolumn{1}{|c|}{ Outputs } \\
\hline $\begin{array}{l}\text { Número de universidades posicionadas en el } \\
\text { ARWU } 20\end{array}$ & $\begin{array}{l}\text { Exportación de productos de alta tecnología } \\
\text { (US\$ a precios actuales) }\end{array}$ \\
\hline $\begin{array}{l}\text { Número de universidades posicionadas en el } \\
\text { ARWU } 100\end{array}$ & Solicitudes de patentes, residentes \\
\hline $\begin{array}{l}\text { Número de universidades posicionadas en el } \\
\text { ARWU } 200\end{array}$ & Crecimiento del PIB (\% anual) \\
\hline $\begin{array}{l}\text { Número de universidades posicionadas en el } \\
\text { ARWU } 300\end{array}$ & PIB (US\$ a precios actuales) \\
\hline $\begin{array}{l}\text { Número de universidades posicionadas en el } \\
\text { ARWU 400 }\end{array}$ & PIB per cápita (US\$ a precios actuales) \\
\hline $\begin{array}{l}\text { Número de universidades posicionadas en el } \\
\text { ARWU 500 }\end{array}$ & \\
\hline $\begin{array}{l}\text { Gasto por alumno, nivel terciario (\% del PIB } \\
\text { per cápita) }\end{array}$ & \\
\hline $\begin{array}{l}\text { Gasto público en educación, total (\% del gasto } \\
\text { del gobierno) }\end{array}$ & \\
\hline Gasto público en educación, total (\% del PIB) & \\
\hline Gasto en investigación y desarrollo (\% del PIB) & \\
\hline
\end{tabular}

Fuente: Elaboración propia con información del ARWU (2017) y el Banco Mundial (2017).

Los primeros seis indicadores fueron explicados en la tabla 1, y los cuatro indicadores restantes provienen del Banco Mundial (2017). A continuación se describirá cómo están compuestos: 
- El gasto por alumno, a nivel terciario (\% del PIB per cápita), se refiere al gasto público corriente en educación por estudiante, dividido entre la cantidad total de estudiantes por nivel terciario, como porcentaje del PIB per cápita (Banco Mundial, 2017). Por otro lado, la UNESCO (2005) lo define como el indicador que representa el gasto en los servicios educativos públicos por alumno de los distintos niveles de escuela pública. Además, enfatiza que es necesario expresarlo como proporción del PIB per cápita ya que es una medida del esfuerzo de un país para otorgar educación a sus habitantes y hace posible realizar comparaciones internacionales.

- Así pues, la magnitud del gasto público resultará de dividir los gastos totales en educación según el nivel de referencia (nivel superior para efectos de este artículo) entre la matrícula que atienden las escuelas públicas en ese nivel. El conocer los efectos del gasto por alumno a nivel terciario está directamente relacionado con el nivel educativo ya que mientras más alto sea el nivel, mayor será la inversión requerida.

- En relación, el gasto público en educación como porcentaje del gasto total del gobierno corresponde al gasto total en educación, expresado como porcentaje del gasto total del gobierno en todos los sectores en un año determinado (Banco Mundial, 2017). Igualmente, la UNESCO (2005) lo describe como el gasto total que realizan los gobiernos federal, estatales y municipales, y los particulares, en educación, tecnología y preservación de la cultura en un determinado año fiscal. Sirve para mostrar en forma agregada el total de recursos que un país ejerce en la atención de la demanda educativa y en el fomento a la investigación, cultura y deporte. Dicha forma es presentada en términos del PIB, considerado como un indicador que permite establecer comparaciones con los gastos en educación de otros países.

- El gasto público total en educación comprende todo aquel que está integrado en cuentas corrientes y capital que se destinan a la educación, y se expresa como un porcentaje del Producto Interno Bruto (PIB) en un año determinado, en este caso los años 2012, 2013 y 2014 (Banco Mundial, 2017). A su vez, la UNESCO (2005) lo define como un indicador que muestra el total de recursos que destina cada nación para atender la demanda educativa en todos los niveles y modalidades de servicio, así como para llevar a cabo las actividades de investigación, cultura y deporte.

- Por último, el gasto en Investigación y Desarrollo (I+D) es el total de gastos corrientes y de capital invertido en el trabajo creativo, realizado como parte de un todo para aumentar los conocimientos y hacer uso de estos para nuevas aplicaciones. "El área de I+D abarca la investigación básica, investigación aplicada y desarrollo experimental" (Banco Mundial, 2017).

Al igual que algunas de las variables de entrada, las variables de salida u outputs fueron obtenidas a partir de datos del Banco Mundial (2017), de las cuales se derivan los siguientes cinco indicadores:

- Exportaciones de productos de alta tecnología (US\$ a precios actuales), este indicador corresponde a productos que para su obtención fue necesario realizar grandes aportaciones en

Diólo@os

sobre Educación

TEMAS ACTUAL año 12 | número 23 | julio-diciembre 2021 | ISSN 2007-2171 
I+D, entre ellos, destacan los productos procedentes de las industrias aeroespacial, informática, farmacéutica, de instrumentos científicos y de maquinaria eléctrica (Banco Mundial, 2017). O sea, usando un enfoque de bienes y servicios, se mide la diferencia existente entre el gasto en investigación y desarrollo, y el total de las ventas procedentes de dicho gasto.

- Las solicitudes de patentes residentes, son aquellas solicitudes presentadas en todo el mundo a través del procedimiento del Tratado de Cooperación en Materia de Patentes o en una oficina nacional de patentes, por los derechos exclusivos sobre un invento ${ }^{6}$ (Banco Mundial, 2017). Se eligió dicha variable debido a la relación que existe entre el aumento de solicitudes de patentes como referente del desempeño de la innovación tecnológica en un país. Lo anterior, de acuerdo al CAF (2016), el cual señala que se establece una relación entre la innovación tecnológica, medida en solicitudes de patentes y la población que está relacionada con las actividades de innovación. A su vez, señala la importancia de las solicitudes de patentes en comparación con la dinámica de las economías nacionales.

- En el crecimiento del PIB (\% anual), los agregados están expresados en dólares estadunidenses, a precios constantes del año 2010.

- El indicador de PIB (US\$ a precios actuales) corresponde a la suma del valor agregado bruto de todos los productores residentes en la economía, más todo impuesto a los productos, menos los subsidios no incluido en el valor de los productos.

- Por otra parte, el PIB per cápita (US\$ a precios actuales), es el producto interno bruto dividido entre la población a mitad del año. Así pues, el PIB es la suma del valor agregado bruto de todos los productores residentes en la economía, más todo impuesto a los productos. Se calcula sin hacer deducciones por depreciación de bienes manufacturados o por agotamiento y degradación de recursos naturales (Banco Mundial, 2017).

Debido a que tan solo 43 países se encuentran posicionados dentro del ARWU en los periodos 2012, 2013 y 2014, el presente estudio se limita espacialmente a los países que se encuentran en la tabla 3.

6 Entiéndase por invento a un producto o proceso que presenta una nueva manera de hacer algo o una nueva solución técnica a un problema. 
Tabla 3. DMU

\begin{tabular}{|l|l|l|l|l|l|}
\hline País & AB & País & AB & País & AB \\
\hline Alemania & AL & España & ES & Nueva Zelanda & NZ \\
\hline Arabia Saudita & AS & Estados Unidos & USA & Países Bajos & PB \\
\hline Argentina & AR & Finlandia & FN & Polonia & PO \\
\hline Australia & AT & Francia & FR & Portugal & PG \\
\hline Austria & AU & Grecia & GR & Reino Unido & RU \\
\hline Bélgica & BE & Hungría & HU & República Checa & RC \\
\hline Brasil & BR & India & IN & República Federal Rusa & RF \\
\hline Canadá & CA & Irán & IR & Serbia & SR \\
\hline Chile & CL & Irlanda & ID & Singapur & SG \\
\hline China & CH & Israel & IS & Sudáfrica & SD \\
\hline Corea del sur & CS & Italia & IT & Suecia & SS \\
\hline Croacia & CR & Japón & JP & Suiza & SZ \\
\hline Dinamarca & DN & Malasia & MA & Turquía & TQ \\
\hline Egipto & EG & México & ME & & \\
\hline Eslovenia & EV & Noruega & NO & & \\
\hline
\end{tabular}

Fuente: elaboración propia con información del ARWU (2017), AB corresponde a la abreviatura de los países.

Se entiende por DMU las entidades donde se toman decisiones, es decir, donde se trasforman insumos en productos o servicios (Chediak, Valencia, 2008: 77). De modo que se asume que los gobiernos de estos 43 países, a partir de su naturaleza (conducir la política interior del Estado en materia de orden público, seguridad ciudadana, servicios sociales y vida económica) son considerados como DMU para efectos de esta investigación.

\section{Metodología}

"La productividad total de factores (PTF) es una medida que forma parte de los elementos que influyen en el crecimiento económico" (Fernández, Almagro, Terán, 2013: 51). No se explica por la cantidad de insumos utilizados, sino que se determina por la eficiencia e intensidad con que se combinan dichos insumos en lo que se denomina como residuo. En otras palabras, las fuentes tradicionales del crecimiento económico son: capital (K) y trabajo (L), pero en estudios recientes se incorporó en la medición de la PTF la contribución de los insumos intermedios que se utilizan en la producción, tales como la energía (E), materiales (M) y servicios (S) (INEGI, 2013).

Con el índice de Malmquist o IM (1953), se hace un acercamiento al crecimiento de la productividad total de los factores (PTF) de una unidad productiva. Igualmente, mediante el IM se puede conocer qué tan eficiente es la DMU, basado en los cambios tecnológicos en el tiempo, 
los cuales se exteriorizan como desplazamientos o cambios de la frontera ${ }^{7}$ mediante la evaluación del comportamiento de inputs y outputs. Por lo cual en este trabajo se hará uso del índice de Malmquist.

Siguiendo el planteamiento anterior, los desplazamientos fuera de la frontera tecnológica indicarán aquellos periodos donde la utilización de recursos resultó menos eficiente, en comparación con las prácticas empleadas en periodos anteriores. Se denomina ineficiencia técnica a aquella distancia entre la frontera y los puntos de producción. "Las funciones distancia o medidas de eficiencia técnica, permiten cuantificar la ineficiencia técnica en un plan de producción, mediante la comparación de las cantidades de insumos y de productos que necesita una unidad que es técnicamente eficiente con los que utiliza una unidad ineficiente" (Martínez, Damián, Brambilla, García, 2013).

Caves, Christensen y Diewert (1982), en su artículo "The Economic Theory of Index Numbers and the Measurement of Input, Output and Productivity", señalan que el IM obtenido con la técnica de Análisis Envolvente de Datos (DEA por sus siglas en inglés), se encarga de definir funciones distancia orientadas a insumos que caracterizan una tecnología por la máxima contracción proporcional posible en el uso de inputs, entretanto los outputs se mantienen constantes, lo cual permite analizar la expansión proporcional de producción dado los inputs empleados.

Una crítica al DEA es que no considera los efectos aleatorios. Pero, ¿qué quiere decir esto?, que todos aquellos acontecimientos que se consideren externos y que puedan ocasionar una desviación de la frontera y afecten el desempeño de una unidad son captados como ineficiencias, lo cual puede o no subestimar la medición (Martínez, Damián, Brambilla, García, 2013).

La utilización del IM guarda propiedades que la hacen atractiva para su implementación: primero, se basa en una técnica no paramétrica, es decir, no requiere especificar una forma funcional ni estimar sus parámetros; segundo, permite definir la frontera tecnológica o las mejores prácticas, a partir de las consideradas en la muestra y comparar las observaciones de cada DMU con la frontera tecnológica.

Lo anterior, hace de los resultados del análisis una aproximación más cercana a la realidad, debido a que el comparativo entre DMU es entre unidades que guardan las mismas condiciones, y no se realiza una comparación con DMU ideales.

El IM evalúa el efecto de eficiencia y el efecto de frontera que experimentan las DMU para determinar el efecto de eficiencia denominado catch up (Vargas, Olivar, Cepeda, 2016), que se traduce en recuperación; es calculado para ver si se experimentó un progreso o decrecimiento en la eficiencia relativa de un periodo 1 al 2 de una DMU, para lo cual se calcula el cociente entre eficiencia con respecto a la frontera del periodo 1. Dicha herramienta fue propuesta por primera vez por Caves, Christensen y Diewert en 1982.

7 Se hace referencia a la convergencia, es decir, a los cambios en la distancia de la frontera, así, un país podría posicionarse sobre la frontera en un periodo, pero no en el siguiente. 
Los IM están basados en el DEA, es decir, se necesita calcular previamente los parámetros de eficiencia para calcular los IM.

$$
I M_{o}\left(x^{t+1}, y^{t+1}, x^{t}, y^{t}\right)=\left[\left(\frac{D_{o}^{t}\left(x^{t+1}, y^{t+1}\right)}{D_{o}^{t}\left(x^{t}, y^{t}\right)}\right)\left(\frac{D_{o}^{t+1}\left(x^{t+1}, y^{t+1}\right)}{D_{o}^{t+1}\left(x^{t}, y^{t}\right)}\right)\right]^{(1 / 2)}
$$

En el IM, un valor mayor que la unidad indica una mejora en la productividad, y cuando es menor que la unidad, evidencia merma de la productividad con respecto al periodo inicial o base. Si es igual a la unidad, muestra una situación de estancamiento productivo (sin cambios). La cual, a su vez, puede ser dividida en otros dos componentes: el cambio en la eficiencia (E) y el cambio tecnológico $(\mathrm{T})$ :

$$
I M_{o}\left(x^{t+1}, y^{t+1}, x^{t}, y^{t}\right)=[\underbrace{\frac{D_{o}^{t+1}\left(x^{t+1}, y^{t+1}\right)}{D_{o}^{t}\left(x^{t}, y^{t}\right)}}_{E}] \cdot[\underbrace{\left(\frac{D_{o}^{t}\left(x^{t+1}, y^{t+1}\right)}{D_{o}^{t+1}\left(x^{t+1}, y^{t+1}\right)}\right)\left(\frac{D_{o}^{t+1}\left(x^{t}, y^{t}\right)}{D_{o}^{t+1}\left(x^{t}, y^{t}\right)}\right)}_{T}]^{(1 / 2)}
$$

La primera ratio representa el cambio en la eficiencia técnica (E), y la segunda, el cambio tecnológico o catch up $(\mathrm{T})$.

En el caso de la orientación hacia el input, si el resultado es menor que 1, implicaría una mejora de la productividad en el tiempo. “En el marco de una orientación a los inputs, esto es, el cálculo de la máxima reducción en los insumos para lograr un determinado nivel de productos" (Aguilar-Barceló, Higuera-Cota, 2019: 19). En el caso opuesto, es decir, con orientación hacia el output, es decir, a las salidas, se da cuando es necesario aumentar los outputs en relación con los inputs, y la interpretación sería la siguiente: solo habría mejora en caso de que el resultado fuera mayor a 1. Tanto en el primer caso como en el segundo, si el resultado es exactamente 1, la interpretación será que no ha habido cambios, no se presentan mejoras o empeoramiento de la productividad de un periodo a otro. ${ }^{8}$

El segundo componente mide el cambio en el progreso tecnológico (T). En el caso de la orientación al input, si el resultado es menor que 1 indica progreso tecnológico, mientras que en un proceso enfocado hacia el output, un cambio superior a 1 mostraría progreso tecnológico.

En este trabajo se consideran las funciones distancia orientadas al producto (output), orientación que resulta adecuada ya que los objetivos de los países se traducen en alcanzar los mayores niveles de producto posibles dada la dotación de recursos existente, como en el caso de la educación, pues el objetivo es alcanzar los mayores niveles de crecimiento y desarrollo con los

8 No obstante, existe otra clase de literatura que incluye modelos no orientados, ejemplos de ellos son el de Farrell y Fieldhouse (1962), denominado eficiencia relativa; el de Charnes, Cooper y Rhodes (1978); y el de Coelli, Estache, Perelman y Trujillo (2003), que trabajan con la eficiencia relativa, entre otros. 
actuales inputs, no en lograr un producto determinado con un nivel mínimo de insumos.

\section{Resultados}

En las tablas 4 y 5 se muestran los resultados del cálculo de la eficiencia en los 43 países mediante los índices de Malmquist para el periodo 2012 a 2014, lo cual se consiguió por medio del empleo del software Win4Deap 2.

Tabla 4. Resultados de eficiencia con el índice Malmquist 2012-2014

\begin{tabular}{|c|c|c|c|c|c|c|c|c|c|c|c|}
\hline País & $\begin{array}{l}\text { CE } \\
\text { (1) }\end{array}$ & $\begin{array}{l}\text { CT } \\
(2) \\
\end{array}$ & $\begin{array}{l}\text { CETP } \\
(3) \\
\end{array}$ & $\begin{array}{l}\text { CEE } \\
(4)\end{array}$ & $\begin{array}{l}\text { IM } \\
(5)\end{array}$ & País & $\begin{array}{l}\text { CE } \\
(1)\end{array}$ & $\begin{array}{l}C T \\
(2)\end{array}$ & $\begin{array}{l}\text { ETP } \\
\text { (3) }\end{array}$ & $\begin{array}{l}\text { EE } \\
(4) \\
\end{array}$ & $\begin{array}{l}\text { IM } \\
(5) \\
\end{array}$ \\
\hline$A L$ & 1.033 & 0.861 & 0.994 & 1.04 & 0.89 & IR & 1 & $* * *$ & 1 & 1 & $* * *$ \\
\hline AR & 0.956 & 1.041 & 1 & 0.956 & 0.995 & IS & 1.265 & 0.881 & 1.611 & 0.785 & 1.115 \\
\hline AS & 1 & 0.609 & 1 & 1 & 0.609 & IT & 0.833 & 0.944 & 1 & 0.833 & 0.786 \\
\hline AT & 1.316 & 0.779 & 1 & 1.316 & 1.025 & JP & 0.852 & 1.217 & 1 & 0.852 & 1.037 \\
\hline$A U$ & 0.719 & 0.306 & 0.925 & 0.777 & 0.22 & MA & 1 & $* * *$ & 1 & 1 & $* * *$ \\
\hline $\mathrm{BE}$ & 0.574 & 1.001 & 0.622 & 0.923 & 0.575 & ME & 1 & 1.017 & 1 & 1 & 1.017 \\
\hline BR & 1 & 1.052 & 1 & 1 & 1.052 & USA & 1 & 1.024 & 1 & 1 & 1.024 \\
\hline $\mathrm{CH}$ & 1 & 1.196 & 1 & 1 & 1.196 & NO & 1 & 0.554 & 1 & 1 & 0.554 \\
\hline $\mathrm{CL}$ & 1 & 0.847 & 1 & 1 & 0.847 & NZ & 1 & 0.591 & 1 & 1 & 0.591 \\
\hline CA & 1 & 1.058 & 1 & 1 & 1.058 & PB & 1.702 & 0.806 & 1.548 & 1.1 & 1.372 \\
\hline CR & 1. & $* * *$ & 1 & 1 & $* * *$ & PO & 0.797 & 1.244 & 0.991 & 0.804 & 0.991 \\
\hline CS & 1 & 1.16 & 1 & 1 & 1.16 & PG & 0.575 & 0.602 & 1 & 0.575 & 0.346 \\
\hline $\mathrm{DN}$ & 0.94 & 1.009 & 0.914 & 1.029 & 0.949 & $\mathrm{RC}$ & 1 & 1.023 & 1 & 1 & 1.023 \\
\hline EG & 1 & 0.879 & 1 & 1 & 0.879 & RF & 1 & 1.602 & 1 & 1 & 1.602 \\
\hline ES & 1.098 & 0.964 & 1 & 1.098 & 1.059 & $R U$ & 0.419 & 1.081 & 0.627 & 0.669 & 0.454 \\
\hline EV & 1 & 1.145 & 1 & 1 & 1.145 & SD & 1 & 0.474 & 1 & 1 & 0.474 \\
\hline FN & 1.159 & 0.926 & 0.992 & 1.169 & 1.073 & SS & 1.095 & 0.979 & 1.075 & 1.019 & 1.072 \\
\hline FR & 0.971 & 1.076 & 0.984 & 0.986 & 1.044 & SP & 1 & 1.134 & 1 & 1 & 1.134 \\
\hline GR & 1 & 1.028 & 1 & 1 & 1.028 & SR & 2.452 & 1.4 & 2.452 & 1 & $3.43^{*}$ \\
\hline $\mathrm{HN}$ & 0.73 & 0.189 & 0.873 & 0.836 & 0.138 & SZ & 1.239 & $* * *$ & 1 & 1.239 & $* * *$ \\
\hline ID & 1 & 1.104 & 1 & 1 & 1.104 & TQ & 1 & 1.286 & 1 & 1 & 1.286 \\
\hline IN & 1 & 1.226 & 1 & 1 & 1.226 & & & & & & \\
\hline \multicolumn{7}{|c|}{ Media geométrica } & 0.981 & 0.956 & 1.014 & 0.968 & 0.983 \\
\hline \multicolumn{12}{|c|}{$\begin{array}{l}\text { Nota: Valores superiores a } 1 \text { indican mejora, e inferiores a } 1 \text { deterioro. } \\
\text { CE: Cambio en la eficiencia técnica. CT: Cambio tecnológico. CETP: Cambio en la eficiencia técnica } \\
\text { pura. CEE: Cambio en la eficiencia de escala. IM: Índice de Productividad de Malmquist PTF. } \\
\text { *Datos con valores faltantes. }\end{array}$} \\
\hline
\end{tabular}

Fuente: elaboración propia con información del Banco Mundial y el ARWU (2012, 2013 y 2014). 
En el periodo de análisis, Alemania, Suecia, España, Finlandia, Suiza, Israel, Austria, Países Bajos y Serbia muestran una mejoría en lo concernirte a la eficiencia en el uso de los insumos, que representan $21 \%$ de los países seleccionados. Se muestra que el promedio se encuentra solo un poco por arriba de lo necesario para exponer un resultado positivo (1.017).

Figura 3. Porcentajes de eficiencia 2012-2014

Cambio de eficiencia técnica (CE)

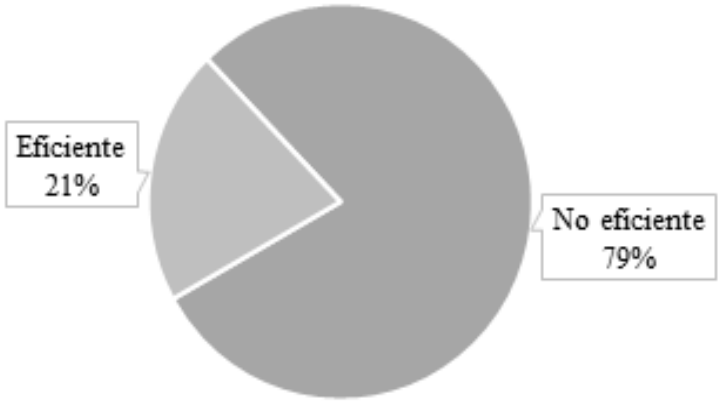

Fuente: elaboración propia, resultados del análisis en el software estadístico Win4Deap 2 (2015).

Al descomponer el índice de eficiencia (figura 3) en el cambio de eficiencia pura y cambio en eficiencia de escala, con valores de 1.014 y 0.968 respectivamente, se puede observar cómo en el primero de estos se presenta una evolución considerada positiva, puesto que los insumos están siendo empleados con mayor eficiencia; sin embargo, el valor de escala se muestra como ineficiente al presentar un valor por debajo del mínimo requerido, es decir, uno.

En lo referente a la productividad, se puede observar cómo 59\% de los países representados en el análisis tienen un valor positivo, destacan China (1.196), Estados Unidos (1.024), Sudáfrica (1.115), Países Bajos (1.372), Rusia (1.602). Por otra parte, en promedio, el índice no tiene un impacto importante sobre el total de los países, pues con el valor de 0.983 se puede considerar que incluso hubo un retroceso. 
Figura 4. Productividad Total de Factores 2012-2014

Cambio total de la productividad del factor (PTF)

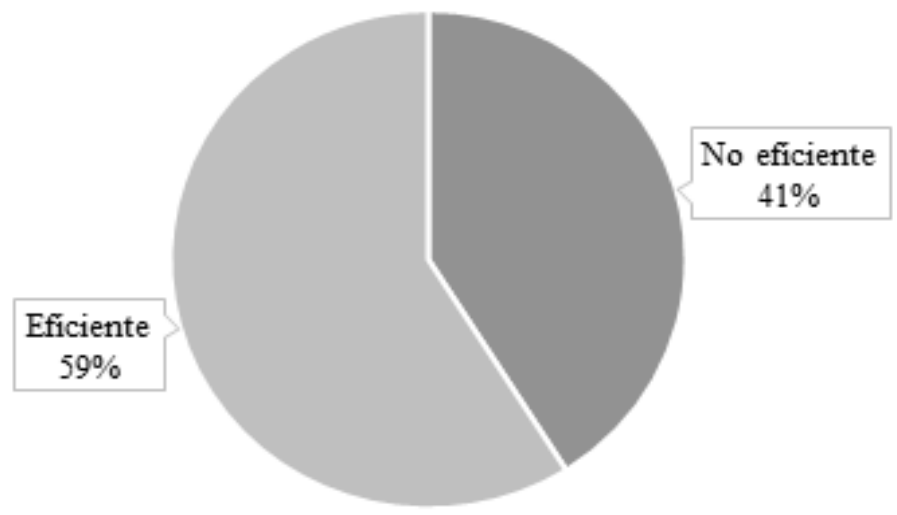

Fuente: elaboración propia, resultados del análisis en el software estadístico Win4Deap 2 (2015).

De igual manera, el cambio tecnológico, en promedio, es ineficiente puesto que al presentar el valor de 0.983 se considera que existe un deterioro en relación con el año anterior. Hungría resulta ser el país más atrasado, con 0.19 , y Rusia en el extremo contrario, con 1.6.

Figura 5. Resultados del índice de Malmquis

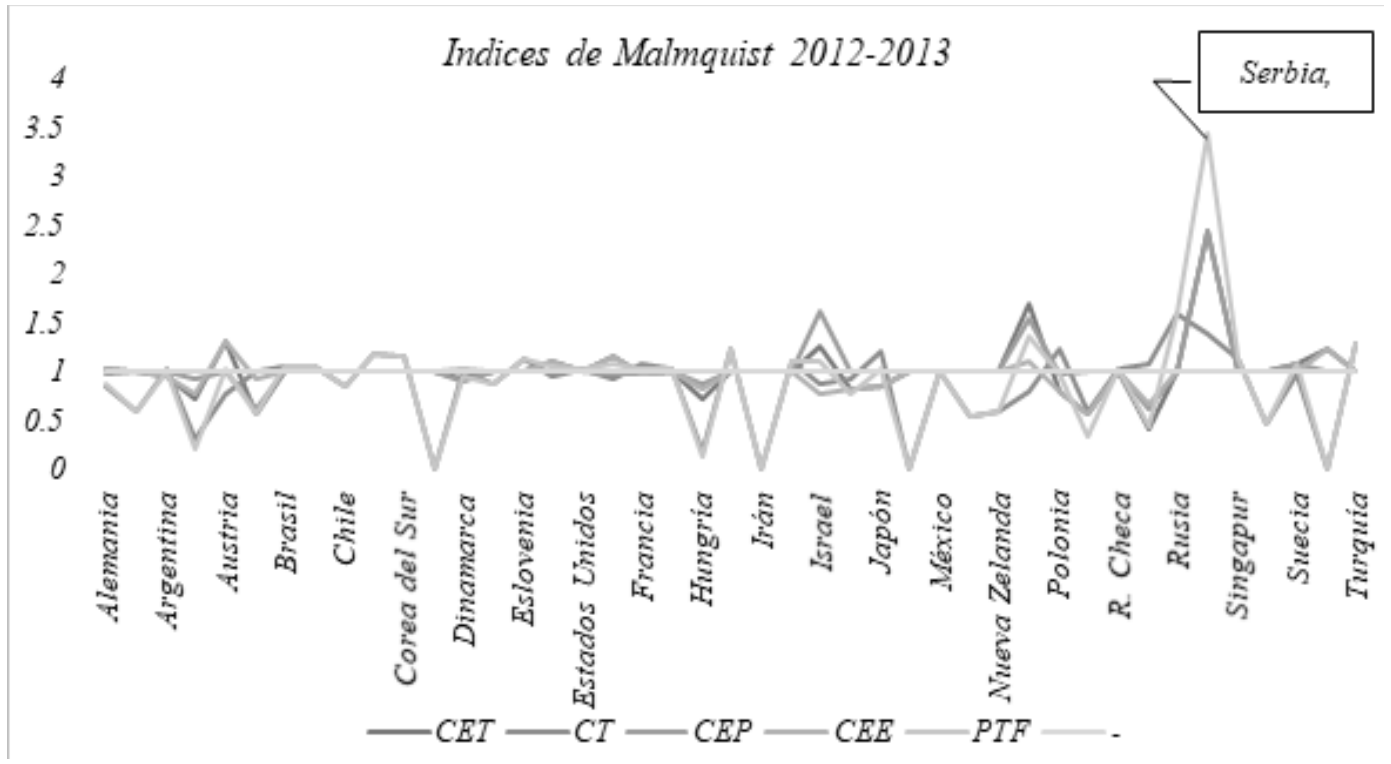

Fuente: elaboración propia, resultados del análisis en el software estadístico Win4Deap 2 (2015). 
Como se puede observar en la figura 5, en general los países tienen una tendencia a generar eficiencia apenas por encima de lo requerido, con un rango de entre 1 y 1.6, con la clara excepción de Serbia, que presenta valores atípicos (esto por una sobrestimación debido a la falta de datos para el año 2012) en el cambio en eficiencia, el cambio en tecnología y el cambio de la productividad total, respectivamente.

En el segundo periodo considerado (de 2013 a 2014), Egipto fue el país que experimentó el mayor incremento de la productividad (véase la tabla 5) al lograr $60.5 \%$, que se debió totalmente a la mejora de la tecnológica ya que presentó un estancamiento de la eficiencia técnica. De esta forma, Egipto mejoró en el periodo 2013-2014 su estructura producto-insumo, lo cual le permitió acercarse a la zona media de la frontera de producción, donde operan los países con las economías desarrolladas. 
Tabla 5. Resultados de eficiencia con el índice Malmquist 2013-2014

\begin{tabular}{|c|c|c|c|c|c|c|c|c|c|c|c|}
\hline País & $\begin{array}{l}\text { CE } \\
(1)\end{array}$ & $\begin{array}{l}C T \\
(2)\end{array}$ & $\begin{array}{l}\text { CETP } \\
\text { (3) }\end{array}$ & $\begin{array}{l}\text { CEE } \\
\text { (4) }\end{array}$ & $\begin{array}{l}\text { IM } \\
(5)\end{array}$ & País & $\begin{array}{l}\text { CE } \\
\text { (1) }\end{array}$ & $\begin{array}{l}C T \\
(2)\end{array}$ & $\begin{array}{l}\text { ETP } \\
\text { (3) }\end{array}$ & $\begin{array}{l}\text { EE } \\
(4)\end{array}$ & $\begin{array}{l}\text { IM } \\
(5)\end{array}$ \\
\hline $\mathrm{AL}$ & 0.767 & 1.355 & 1.026 & 0.747 & 1.04 & IR & 1 & 0 & 1 & 1 & 0 \\
\hline$A R$ & 0.812 & 1.186 & 0.744 & 1.091 & 0.962 & IS & 0.746 & 1.494 & 0.773 & 0.965 & 1.114 \\
\hline AS & 1 & $* * *$ & 1 & 1 & $* * *$ & IT & 0.454 & 1.977 & 0.843 & $0.53^{8}$ & 0.897 \\
\hline AT & 0.593 & 1.714 & 1 & 0.593 & 1.016 & $\mathrm{JP}$ & 0.965 & 1.056 & 1 & 0.965 & 1.019 \\
\hline$A U$ & 1.39 & 3.465 & 1.081 & 1.286 & $4.81 *$ & MA & 1 & 0 & 1 & 1 & 0 \\
\hline $\mathrm{BE}$ & 0.709 & 1.491 & 0.932 & 0.761 & 1.058 & ME & 1 & 1.331 & 1 & 1 & 1.331 \\
\hline BR & 0.571 & 1.719 & 1 & 0.571 & 0.981 & USA & 0.533 & 2.207 & 1 & 0.533 & 1.177 \\
\hline $\mathrm{CH}$ & 1 & 0.953 & 1 & 1 & 0.953 & NO & 1 & 1.054 & 1 & 1 & $1.05^{*}$ \\
\hline $\mathrm{CL}$ & 1 & 0.705 & 1 & 1 & 0.705 & NZ & 1 & 2.556 & 1 & 1 & $2.55^{\star}$ \\
\hline CA & 0.448 & 2.166 & 1 & 0.448 & 0.97 & PB & 0.503 & 2.094 & 0.994 & 0.506 & 1.054 \\
\hline CR & 1 & $* * *$ & 1 & 1 & $* * *$ & PO & 1.048 & 1.029 & 0.975 & 1.075 & 1.079 \\
\hline CS & 1 & 1.291 & 1 & 1 & 1.291 & PG & 0.817 & 1.132 & 0.694 & 1.177 & 0.925 \\
\hline DN & 0.806 & 1.297 & 1.046 & 0.77 & 1.045 & RC & 0.648 & 1.814 & 1 & 0.648 & 1.175 \\
\hline EG & 1 & 1.605 & 1 & 1 & 1.605 & RF & 1 & 0.923 & 1 & 1 & 0.923 \\
\hline ES & 0.545 & 1.406 & 0.877 & 0.622 & 0.767 & $R U$ & 0.697 & 1.524 & 1.085 & 0.642 & 1.062 \\
\hline EV & 0.77 & 1.842 & 1 & 0.77 & 1.418 & SD & 0.504 & 4.019 & 0.509 & 0.989 & $2.02 *$ \\
\hline $\mathrm{FN}$ & 0.8 & 1.293 & 1.026 & 0.78 & 1.034 & SS & 0.784 & 1.303 & 1.08 & 0.726 & 1.022 \\
\hline FR & 0.817 & 1.453 & 1.423 & 0.574 & 1.187 & SP & 1 & 2.65 & 1 & 1 & $2.65 *$ \\
\hline GR & 1 & 1.68 & 1 & 1 & 1.68 & SR & 0.41 & 1.938 & 0.624 & 0.658 & 0.795 \\
\hline $\mathrm{HN}$ & 0.922 & 1.261 & 1.067 & 0.864 & 1.163 & $S Z$ & 1 & 0.096 & 1 & 1 & $0.09 *$ \\
\hline ID & 1 & 1.16 & 1 & 1 & 1.16 & TQ & 1 & 4.452 & 1 & 1 & $4.45^{*}$ \\
\hline IN & 1 & 0.792 & 1 & 1 & 0.792 & & & & & & \\
\hline \multicolumn{7}{|c|}{ Media geométrica } & 0.808 & 1.573 & 0.961 & 0.841 & 1.269 \\
\hline
\end{tabular}

Nota: Valores superiores a 1 indican mejora, e inferiores a 1 deterioro.

CE: Cambio en la eficiencia técnica CT: Cambio tecnológico. CETP: Cambio en la eficiencia técnica pura. CEE:

Cambio en la eficiencia de escala. IM: Índice de Productividad de Malmquist PTF.

*Datos con valores faltantes.

Fuente: elaboración propia con información del Banco Mundial y ARWU (2013) y (2014). 
Por otra parte, Brasil sufrió la mayor caída (decremento) de la productividad al reflejar un descenso de $30 \%$, debido al desaceleramiento del progreso tecnológico. Lo cual también puede ser atribuible al drástico descenso en el crecimiento económico de esos años, debido a que pasó de tener una tasa de crecimiento anual de 4\% en 2013, a 1.7\% para 2014.

En lo referente a la productividad, se puede observar que $62 \%$ de los países analizados tienen un valor positivo, entre los que destacan Alemania (1.04), Estados Unidos (1.177), Corea del Sur (1.291), Dinamarca (1.045), Francia (1.187), Países Bajos (1.054), Suecia (1.022). Por otra parte, en promedio el índice no tiene un impacto importante sobre el total de los países, pues con el valor de 0.983 se puede considerar que incluso hubo un retroceso.

Es importante señalar que el IM también puede ser influenciado por otros factores, tales como los tipos de productos e insumos empleados en sus cálculos, la calidad de la información colectada en el periodo analizado, así como por el nivel de desarrollo del país donde se realiza el estudio (Jan, 2011); por lo cual, las comparaciones entre grupos de países o regiones diferentes deben ser tomadas con la debida precaución.

\section{Conclusiones}

A través de este artículo se dio respuesta al siguiente planteamiento: ¿cuáles son los efectos de la educación superior sobre la productividad de los países? Encontramos que al analizar un total de 43 países, acudiendo al Análisis Envolvente de Datos (DEA) y a la descomposición de factores del Índice de Malmquist, en su mayoría los países muestran un desempeño deficiente; sin embargo, el que $41 \%$ de los países analizados sea eficiente es un indicador positivo para considerar a la educación superior como un elemento clave a la hora de evaluar la productividad de los países.

A partir de este análisis se encontró que si los países quieren elevar sus niveles de productividad es necesario que sigan las pautas de Alemania, Suecia, España, Finlandia, Suiza, Austria y Países Bajos.

Considerando que se tomaron en cuenta elementos como el posicionamiento de los países dentro del Ranking Académico de las Universidades del Mundo, las exportaciones de productos de alta tecnología, solicitudes de patentes y el PIB, entre otros, es importante señalar que a lo largo de la realización del artículo existieron limitaciones de información y, aunque ha sido posible establecer un desempeño que evidencia una deficiente gestión de los recursos educativos asignados para alcanzar el mejoramiento de la productividad en el ámbito de cada nación, aún quedan muchas interrogantes por responder, como cuál de los elementos antes mencionados tiene mayor influencia a la hora de evaluar la productividad.

Cabe hacer énfasis en que el presente trabajo se trata de un análisis parcial de productividad, debido a que el índice de Malmquist contempla un análisis de PTF. Además, el ARWU en sí posee detractores pues su función es subjetiva respecto a las necesidades planteadas al

Diálo@os sobre Educación año 12 | número 23 | julio-diciembre 2021 | ISSN 2007-2171 
momento de su creación, como el hecho de que para China era necesario conocer su brecha de investigación respecto a otros países. Igualmente, no se incluyeron las prioridades por nación.

En cuanto a las líneas de investigación futura, es de suma importancia incorporar variables asociadas a la competitividad y la calidad de vida. Por último, sería de gran importancia considerar los esfuerzos individuales de España, Finlandia, Suiza, Israel, Austria y Países Bajos, con la finalidad de conocer a detalle las estrategias y políticas desplegadas por estos países, de modo que los resultados obtenidos fueran más concluyentes.

\section{Referencias}

Aguilar-Barceló, J.; F. Higuera-Cota (2019). Los retos en la gestión de la innovación para América Latina y el Caribe: un análisis de eficiencia. Revista de la CEPAL, (127), 7-26.

Ardila, R. (2003). Calidad de vida: una definición integradora. Revista Latinoamericana de Psicología, 35(2), 161-164.

Banco Mundial (2017). Micro datos: índice de datos. Datos de libre acceso del Banco Mundial. México. https://databank.bancomundial.org/databases

Beck, U. (1988). ¿Qué es la globalización?: Falacias del globalismo, respuestas a la globalización. Buenos Aires: Paidós.

CAF (2016). Iniciativa regional de patentes tecnológicas para el desarrollo: indicadores de la innovación tecnológica de los países.

Carro, R.; D. González (2012). Productividad y competitividad. Buenos Aires: Universidad Nacional de La Plata.

Caves, D.; L. Christensen; W. Diewert (1982). The Economic Theory of Index Numbers and the Measurement of Input, Output and productivity. Econométrica, 50(6), 1393-414.

Charnes, A.; W. Cooper; E. Rhodes (1978). Measuring the Efficiency of Decision-making Units. European Journal of Operational Research, (2), 429-444.

Chediak, P.; L. Valencia (2008). Metodología para medir la eficiencia mediante la técnica del análisis envolvente de datos. Vector, (5), 70-81.

Coelli, T.; A. Estache; S. Perelman; L. Trujillo (2003). Una introducción a las medidas de la eficiencia para reguladores de servicios públicos y de transporte. Colombia: Banco Mundial/Alfaomega.

Delors, J. (1996). La educación encierra un tesoro. Madrid: Santillana.

Falicov, E.; S. Lifzyc (2002). Sociología. Buenos Aires: Aique.

Fernández, R.; F. Almagro; J. Terán (2013). Un análisis de la productividad total de factores ampliada en la industria manufacturera de México 2003-2010. Investigación Administrativa, (112), 51-63. 
Farrell, M.; M. Fieldhouse (1962). Estimating Efficient Production Function under Increasing Returns to Scale. Journal of the Royal Statiscal Society, 125(2), 252-67.

García, E. (2006). Las competencias del profesor en la sociedad del conocimiento. Santo Domingo: Búho.

Giddens, A. (1999). Sociología. Madrid: Alianza Universidad Textos.

Griffith, R.; S. Redding; J.Van Reenen (2004). Mapping the Two Faces of R\&D: Productivity Growth in a Panel of OECD Industries. The Review of Economics and Statistics, 86(4), 883-95.

Grimson, A. (2008). Diversidad y cultura: reificación y situacionalidad. Tabula Rasa, (8), 45-67. http://www.scielo.org.co/pdf/tara/n8/n8a03.pdf

Guerrero, J.; M. Faro (2012). Breve análisis del concepto de Educación Superior. Alternativas en Psicología, (27), 34-41.

INEGI (1996). El ABC de los indicadores de la productividad. Aguascalientes: INEGI.

INEGI (2013). Sistema de cuentas nacionales de México, fuentes y metodologías: productividad total de los factores-modelo de Klems. México: INEGI.

Kantor, J. (1990). La evolución científica de la psicología. Trillas.

León, A. (2007). Qué es la educación. Educere, La Revista Venezolana de Educación, 11(39), 595-04. http://www.saber.ula.ve/bitstream/handle/123456789/20200/articulo2.pdf

Malmquist, S. (1953). Index Numbers and Indifference Surfaces. Trabajos de Estadística, 4(2), 209-42.

Martínez-Damián, M.; J. Brambila-Paz; R. García-Mata (2013). Índice de Malmquist y productividad estatal en México. Agricultura, Sociedad y Desarrollo, 10(3), 359-69.

Mertens, L. (1999). La medición de la productividad como referente de la formación-capacidad articulada con el aprendizaje organizacional: una propuesta metodológica. https://www.oitcinterfor.org/sites/default/files/edit/docref/medicion capacitacion.pdf

Morales, C.; A. Masis (2014). La medición de la productividad del valor agregado: una aplicación empírica en una cooperativa agroalimentaria de Costa Rica. Tec Empresarial, 8(2), 41-49.

Salmi, J.; A. Saroyan (2007). League Tables as Policy Instruments: Uses and Misuses. Higher Education Managment and Policy, 19(2), 1-38.

Shanghai Ranking Consultancy (2017). Academic Ranking of World Universities. http://www.shanghairanking.com/arwu2017.html

Tedesco, J. (2002). Educación y globalización: los desafíos para América Latina. Revista de la CEPAL, (76), 55-69.

Tylor, E. (1871). Teoría del nacionalismo performativo. El Viejo Tipo, (39),19-25.

UNESCO (2005). Panorama educativo 2005: progresando hacia las metas. Santiago: UNESCO. UNESCO (2011). Clasificación internacional normalizada de la educación. Montreal: JC Nicholls. UNICEF (2000). El derecho a la educación: desde el marco de la protección integral de los derechos de la niñez y la política educativa. París/Nueva York: UNICEF. 
Vargas, J.; G. Olivar; E. Cepeda (2016). Malmquist histórico y de pronóstico con series de tiempo como modelo temporal de análisis. Revista Información Tecnológica, 27(3), 141-149.

Villaseñor, J.; C. Moreno; J. Flores (2015). Perspectivas actuales sobre los rankings mundiales de universidades. Revista de la Educación Superior, 3(175), 41-67. 